\title{
Assessing Decision-Making Capacity After Brain Injury:
}

A Phenomenological Approach

\section{$\underline{\text { Authors }}$}

\section{Gareth S Owen}

Contact: Department of Psychological Medicine, Institute of Psychiatry, Weston Education Centre, Cutcombe Rd, London SE5 9RJ; Telephone: 0207848 5479; email: gareth.1.owen@kcl.ac.uk; Fax:02078485408.

Gareth S Owen is clinical senior lecturer at the Institute of Psychiatry, King's College London and a consultant psychiatrist at the South London and Maudsley NHS Foundation Trust. He has scholarly interests in psychiatry and law as well as psychopathology and phenomenology. Some of his recent publications are "Temporal Inabilities and Decision-Making Capacity in Depression" in Phenomenology and the Cognitive Sciences and "Mental Capacity and Decisional Autonomy: An Interdisciplinary Challenge" in Inquiry.

Fabian Freyenhagen

Contact: University of Essex, School of Philosophy and Art History, Wivenhoe, Park, Colchester CO4 3SQ; Telephone: 01206872713; email: ffrey@essex.ac.uk.

Fabian Freyenhagen is Reader in Philosophy at the University of Essex and coInvestigator on the Essex Autonomy Project. His work on autonomy and mental capacity has been published in Inquiry, Phenomenology and Cognitive Sciences, and International Journal of Law in Context. He is the author of Adorno's Practical Philosophy: Living Less Wrongly (Cambridge University Press, 2013).

\section{Wayne Martin}

Contact: University of Essex, School of Philosophy and Art History, Wivenhoe, Park, Colchester CO4 3SQ; Telephone: 01206873405; email: wmartin@essex.ac.uk.

Wayne Martin is Principal Investigator on the Essex Autonomy Project, an AHRC funded research project concerned with the ideal of self-determination in the practice and regulation of care. Recent publications include "An Unblinkered View of Best Interests" in the British Medical Journal and "Mental Capacity and the Applied Phenomenology of Judgement" in Phenomenology and the Cognitive Sciences. 
Assessing Decision-Making Capacity After Brain Injury:

A Phenomenological Approach

\section{$\underline{\text { Abstract: }}$}

The assessment of decision-making capacity in patients with brain injuries presents a range of clinical and legal challenges. Existing guidance on the conduct of such assessments is often generic; guidance specific to patients with brain injury is sparse and coarse-grained. We report on an interview-based study of decision-making capacity in patients suffering from acquired brain injury and organic personality disorder. We identify challenges associated with the assessment of DMC in this patient population, review three bodies of relevant research from cognitive neuropsychology and neurophysiology, and draw on phenomenological analysis to identify three distinct abilities that play a role in decision-making, but which can be compromised in patients with organic personality disorder. We address the challenge of translating clinical findings into legally attestable results.

\section{$\underline{\text { Keywords: }}$}

Mental Capacity, Competence, Organic Personality Disorder, Acquired Brain Injury, Frontal Lobe Syndrome, Phenomenology, Impulsiveness, Awareness of Deficit, Metacognition, Somatic Marker Hypothesis, Environmental Dependency Syndrome 


\section{§1 The Challenge: Assessing DMC in ABI and OPD}

Patients with acquired brain injury (ABI) present special challenges in the assessment of decision-making capacity (DMC) or "competence." We shall here be particularly concerned with the subset of ABI patients who have suffered damage to the frontal lobe and acquired the clinical syndrome of "organic personality disorder" (OPD) sometimes referred to as "the frontal lobe syndrome" and made famous by the case of Phineas Gage. Patients with OPD undergo significant alterations of the habitual patterns of premorbid behavior; the question often arises as to whether a person with such an injury retains $\mathrm{DMC}$, for example in matters pertaining to health care or management of personal finances.

The assessment of DMC is governed by legal standards that are themselves grounded in case law and statute. The details of these standards vary from one jurisdiction to another, albeit in the context of a significant degree of overlap and consensus. In what follows we shall take our orientation from the Mental Capacity Act (England and Wales), 2005 (MCA), which has become a point of reference internationally. The MCA standard can itself been seen as a close variant on the standard of competence for treatment decisions distilled by Grisso and Appelbaum on the basis of a systematic study of case law in the USA (Grisso and Appelbaum 1998).

Capacity assessors dealing with this patient population must navigate a number of obstacles. Clinically, the ABI patient often presents as a kind of "everyman," so deficits associated with the disorder may not manifest themselves in a standard capacity interview. Moreover, patients in this group often manage to mask their own deficits, even from themselves; the assessor must therefore develop strategies for probing beyond the façade. But some of the thorniest difficulties derive from the distinctive legal standing of the concept of DMC, which sets certain rigid 
constraints on both the form and content of the assessment. Legally, the concept of $\mathrm{DMC}$ is a binary concept; moreover, the law requires that an assessment of DMC be decision-specific (MCA s.2; Grisso and Appelbaum 1998). Taken together, these two legal commitments mean that the assessor must reach an up-or-down judgment regarding a patient's capacity to make a particular decision at a particular time.

In arriving at a judgment of the requisite form, the assessor faces two problems. First, many of the behavioral traits associated with OPD manifest themselves on a continuum; they are differences of degree rather than of kind. Where should the assessor "draw the line" so as to demarcate DMC? Call this the continuum problem. The second problem is related. That continuum itself exhibits considerable overlap with the healthy population, where the trait is not generally taken to compromise DMC. Call this the overlap problem. We can illustrate both problems with reference to impulsivity, a trait that is often exhibited in this patient population. Impulsivity is itself a trait that varies by degree: some patients are more impulsive than others. It is also a trait that is well-represented in the healthy population, where it is not generally thought to compromise DMC. In order to resolve the continuum problem, we need to find a principled way of distinguishing competent from incompetent impulsivity. In addressing the overlap problem we must find a way of distinguishing pathological forms of impulsivity from the run-of-the-mill impulsivity that most decision-makers exhibit at one time or another.

A further set of constraints derives from the legal requirement to avoid any outcome test for DMC. One of the animating principles of the MCA is that "A person is not to be treated as unable to make a decision merely because he makes an unwise decision" (MCA, §1.3). But in ABI, it is often the fact of unwise decision-making that is the salient factor and overwhelming concern. In a characteristic scenario, a 
patient faces the prospect of a large financial settlement following an accident. The family, advisors, and the court are rightly concerned that the money will be squandered, leaving the patient without the long-term support that $\mathrm{s} /$ he requires. But in order to adhere to the principles of the MCA, the danger of a poor outcome cannot itself warrant a finding of incapacity. The assessor must instead operate with a functional test for capacity, drawing on the legal definition of DMC as comprising the abilities to understand and retain information relevant to the decision, to use and weigh that information in reaching a decision, and to communicate a choice (MCA, §3.1). (Grisso and Appelbaum's list of abilities is similar: to understand, appreciate, reason and express a choice; Grisso and Appelbaum 1998, 1-2.)

This in turn has bearing on the broadest legal constraint: any finding as to DMC must be legally attestable. The presence or absence of DMC has significant consequences for a patient's legal rights. A patient found to lack DMC may lose the right to refuse medical treatment, for instance, or to enter into certain contracts; he may lose effective control over his personal finances or place of residence. Any finding regarding DMC must accordingly be evidence-based and articulable in a form that can, if necessary, be presented and defended in court. Here it is important to appreciate that the legal standard is itself defined in essentially psychological terms in the MCA, the abilities to understand, retain, use and weigh information. This means that neurophysiological evidence can itself bear only indirectly on a finding of capacity; ultimately the assessor must determine which among the psychological abilities have been compromised by the patient's injuries. But these abilities are described only in very abstract terms in statute and case law, and not in terms that suggest any obvious cut-off point at which the limit of DMC should be established. We should therefore not be surprised to discover that legal cases involving disputed 
DMC in ABI can become protracted, with conflicting expert testimony and further appeals (see, notably, Masterman-Lister v. Brutton and Co, 2002).

In what follows we report on an interview-based study of decision-making in a group of patients with $\mathrm{ABI}$ and OPD. We begin $(\S 2)$ with a review of three recent research paradigms that have generated important results as regards the distinctive patterns of deficit associated with ABI. $\S 3$ describes the parameters of our study. In $\S 4$ we draw on data from the study to identify an obstacle in "translating" insights from the recent research paradigms into strategies for the assessment of DMC in ABI. In $\S 5$ we draw on an insight from Lhermitte's description of environmental dependency syndrome, together with further data from our study, in order to overcome this obstacle. In $\S 6$ we identify a cluster of abilities that are both (a) required for DMC and (b) compromised in some patients with $\mathrm{ABI}$ and OPD. We propose a strategy for resolving the continuum and overlap problems.

Before proceeding further, two clarificatory points are in order. We note first that the definition of mental incapacity in the MCA combines two conceptually discrete elements. Our concern here is with the functional component of the test, which analyses DMC into the four abilities elaborated in MCA s. 3(1). But the MCA also includes a broad diagnostic test: lack of decision-making abilities is tantamount to mental incapacity under the Act only insofar as the patient's inabilities are the result of "an impairment of or disturbance in the functioning of the mind or brain." (MCA s.2(1)). The diagnostic test raises a number of difficulties of its own (Martin 2015). These are not our concern here. Our focus is on the application of the functional component of the DMC standard, which is common to the MCA and many other legal standards. 
The second clarification concerns an objection that has sometimes been leveled against the standard of incapacity found in the Grisso and Appelbaum test, in the MCA and in similar provisions of law in other jurisdictions. In particular, it has been argued that these standards focus too narrowly on specifically cognitive abilities (understanding, retention, reasoning, etc.), to the exclusion of other factors that play a significant role in human decision-making. (For a sampling of this discussion, see Tan 2006, Charland 2006, Craigie 2009.) The present study bears only indirectly on this debate. It is not our purpose here to assess the adequacy of existing legal standards of mental capacity; our purpose rather is to consider how those standards can best be applied to patients with OPD consequent upon brain injury. As we shall see, however, consideration of this matter helps bring into view a variety of ways in which legal standards like the MCA themselves implicitly incorporate requirements that are not narrowly cognitive in nature.

\section{§2 Review: Existing Research Results Regarding Deficit in ABI}

We found only a small literature devoted to the specific problem of capacity assessment in the target group. Some papers rehearse current anatomy, physiology and neuropsychology of the frontal lobes and outline existing legal standards for decision-making abilities (Reid-Proctor et al. 2001; Schindler et al. 1995). Freedman et al. (1991) provide some practical guidelines for assessing DMC in patients with frontal dysfunction. They stress the importance of looking for dissociations between what the patient may report and their actual behavior, and warn that competence can be threatened not merely by the absence of knowledge, but by deficits in the "awareness of the meaning of knowledge and the judgment to act accordingly" (p. 
206). Whilst giving us some leads on what is relevant to capacity assessment in such patients, much is left unspecified.

By contrast, the broader research literature on brain injury is vast, and reaches back about two centuries. But as Dunn et al. (2007) have remarked, "a bridge remains to be built that would link the neuroscience and other decision-making literature to the empirical bioethics literature on decision-making capacity" (p. 341). Our aim here is to contribute to the construction of such a bridge. We begin by surveying three prominent strands in recent research regarding forms of deficit in ABI.

One approach to the measurement of deficit in ABI focuses on lack of awareness of deficit, as documented by "discrepancy scores." This research is largely based on questionnaires, with both patients and informants providing numerical ratings of the patient's abilities in a variety of categories. Discrepancies between the self-assessment and the assessment provided by informants is quantified. Research studies of this format have now been conducted across a wide variety of abilities (cognitive, emotional, social, etc.) and a consistent pattern emerges. Patients with $\mathrm{ABI}$ characteristically rate their own abilities higher than do the informants, and the discrepancy scores are on average higher in the population of patients with $\mathrm{ABI}$ than in the population of healthy research subjects (Prigatano 1992; Bach and David 2006). Using this methodology, one quantitative measure of lack of self-awareness can be produced. Lack of awareness of deficit is potentially relevant to DMC, particularly in contexts where it threatens to undermine understanding of diagnosis and/or the ability to use and weigh (or appreciate) information pertaining to treatment options.

In a second research approach, the central conceptual construct is the notion of metacognition, a term that has been variously defined. The strategy here is to focus on a particular higher cognitive function: the ability to think about cognitive 
processes, whether one's own of those of others. Consider an example. I need to buy a car, and have limited funds for the purchase. One thing I know about myself is that I am prone to enthusiasm when seeing a sleek sports car, even though what I really need is a rather more mundane and affordable sedan. I also know that car salesmen will be sensitive to this form of enthusiasm, and may try to exploit it in making a sale. So I take along a trusted and sober friend to the car lot in order to reduce the risk. This is an example of putting metacognition to work in decision-making. I have the ability to think about my own cognitive processes, and about those of others, and I can then make use of this information in trying to decide effectively. Metacognition is itself closely related to the ability that has been described as "theory of mind," the ability to attribute mental states to myself and to others. A second body of descriptive research has shown that metacognition can be impaired in $\mathrm{ABI}$, and that this deficit itself lies at the root of other difficulties faced by the patient (lack of empathy, lack of social sensitivity, etc.) (Hanten et al. 2000; Ownsworth and Fleming 2005; Ciurli et al. 2010). This second research paradigm partly intersects with the first, insofar as awareness of one's own psychological deficits would itself seem to presuppose a form of metacognition: the ability to think about one's own mental (dis)abilities.

These two research approaches can be described as psychological, making use of quantifiable variables in behavior and cognition. A third research approach has focused on neurophysiological deficits in ABI. The landmark work here is that of Bechara and Damasio, which makes use of neurophysiological monitoring of research subjects performing the so-called "Iowa Gambling Task." Bechara and Damasio have advanced what they call the 'Somatic Marker Hypothesis': the hypothesis that patients with certain forms of frontal lobe damage suffer a very specific physiological deficit, the inability to generate "anticipatory skin conductance resonances" (aSCRs). 
Whereas healthy subjects performing the Iowa Gambling Task develop aSCRs as they gain familiarity with the rules of the game, ABI patients studied by Bechara and Damasio uniformly fail to generate any aSCRs, and this failure is associated with poor performance in the game itself. Although their data is behavioral and physiological, Bechara and (particularly) Damasio have offered a psychological interpretation of the deficit. Damasio argues that aSCRs play an important role in the decision-making of healthy subjects by generating bodily sensations which themselves provide a form of emotional "biasing" of some options over others. Bechara and Damasio (and colleagues) hypothesize that ABI patients who lack aSCRs fail to experience a range of emotional responses that could otherwise serve to guide their decision-making (Bechara et al. 1994; 1996; 1998; 1999; 2005; Damasio 1994).

These three research traditions have each in their way been fertile, and have advanced our understanding of the forms of deficit associated with ABI. But in applying these research results to the task of capacity assessment, it is important to remember that these research paradigms characteristically focus on the distribution of traits and behaviors across a research sample. The results are therefore validated as statistically significant variations and correlations at the level of populations. But in juridical reasoning, statistical information about a population can never of itself suffice; the concern of the law is with individuals. Just as the criminal lawyer cannot build a murder case on statistical information about the prevalence of homicide in the population to which the accused belongs, so the juridical assessment of DMC cannot be satisfied by evidence showing a pattern of impaired metacognitive abilities in patients who have suffered frontal lobe injuries. In order to meet the constraint of legal attestability, research of this kind must be translated into strategies for 
investigating the particular abilities manifested by an individual patient at the material time.

\section{§3 Study Design}

The methodology of the study was a principled hybrid, integrating phenomenological and clinical elements with standard techniques from qualitative interview research. The core strategy involved a technique of second-person hermeneutic phenomenology that we have developed and reported in other research (Owen et al., 2015). A series of open-structured interviews were used to gain insight into the texture of the patient's experience of making decisions under conditions of neuropsychiatric disorder. The interviews were recorded and transcribed and subsequently 'coded' using line-by-line gerundive coding techniques to help generate broader categories. The recordings, coded transcripts and contextual information were then submitted to close interpretative scrutiny. We did not assume that the subjects' reports could be taken at face-value. Instead we used the data from the interviews as evidence from which hypotheses could be generated; we then probed these hypotheses both in subsequent interviews, using collateral information and in further analysis of recordings and transcripts. We used standard techniques of grounded theory (Charmaz 2006) to assist in the construction of higher-order codes and in the thematisation of our data. The interviews were organized around two decisions: we engaged participants in dialogue about the decision that they had made to participate in the research study itself; we then explored a pending decision they faced concerning treatment options. Excerpts from the interviews cited in this article are drawn from a large corpus of interview data. We used Atlas.ti software to facilitate analysis. 
Sample: Our group of interest were patients with ABI and OPD with treatment decisions to make. We followed the approach of Interpretative Phenomenological Analysis of purposely sampling around six cases for in-depth study of relatively homogenous groups (Smith, Flowers and Larkin 2009). Six patients with acquired brain injury (ABI 1-6) satisfying ICD-10 criteria for organic personality disorder (F07.0) and DSM-5 criteria for Personality Change due to acquired brain injury (310.1) participated in the study. All patients were recruited from a pool of people in contact with neurorehabilitative or psychiatric services in the UK.

Patients were selected who demonstrated the mental disorder without comorbid psychiatric or neurological illness or pre-morbid personality disorder. Alcohol or substance misuse was not current for any of the subjects and periods of misuse in the past were not judged to be contributing to the current presentation by the clinical teams or by the researcher. Time since brain injury ranged from 1-19 years.

Each participant was alert, communicative and able to express choices about treatment or research participation. Information about the relevant decisions was easily communicable to the participants and retained for periods sufficient to make discussion about it possible. Apart from one participant who had impaired performance on the WAIS-III and the Rivermead Behavioral Memory Battery, attention, memory and language performances were considered relatively preserved by assessing clinical psychologists. Performance on the Haylings or Brixton tests of executive functioning or the dysexecutive questionnaire (DEX) was impaired in all participants though no individual test was impaired in all subjects. Each subject spoke English as a first language; educational and work histories suggested average pre-morbid intelligence. The neurological histories and neuroimaging reports indicated damage to predominantly the frontal lobes. 
All subjects were men with ages ranging from 25 to 80 . Each one faced significant decisions about treatment that ranged from medication (psychiatric or medical) to therapies (including physio, psychological and occupational) to whether to be receiving home or hospital care. Two participants were detained under the Mental Health Act (1983) - the law in England authorizing compulsory hospital admission and treatment for mental disorder. The other four patients were informal hospital patients.

Interviews: The interviews were conducted by Gareth Owen (Interviewer), who has experience of research interviews involving DMC in people with mental disorder and is a consultant adult and liaison psychiatrist registered in the UK. The interview guide drew upon existing approaches in the literature on assessment of $\mathrm{DMC}$, and was developed by the research team with a view to obtaining rich data relevant to DMC. The guide was piloted with six patients who are not included in the present analysis and modified slightly as a result.

Collateral information was obtained from people the patient trusted (e.g. family members, friends, doctors, psychologists, OTs or nurses who knew them) to gain further perspectives and check against misinterpretations.

Research Ethics: The study received research ethics approval from the South East London Research Ethics Committee. The Mental Capacity Act (2005) rules for research were followed.

\section{$\S 4$ Initial Findings}

In interpreting the interviews, our first step is to draw directly on the results of the three research paradigms reviewed in $\S 2$, above. As we have seen, those research results indicate that $\mathrm{ABI}$ patients characteristically suffer from deficits in awareness- 
of-deficit, in metacognition, and in the signals associated with the so-called 'somatic marker.' Accordingly, we reviewed the transcripts of the interviews with particular attention to these forms of deficit, in order to consider how they manifested themselves in the setting of a capacity assessment interview. The initial results were surprising.

a) Awareness-of-Deficit: Contrary to expectations, the patients in the study exhibited considerable awareness of the deficits associated with their injuries. In three cases the DEX discrepancy scores were very low or even negative, where a negative DEX score indicates that the patient reports greater deficit than an independent rater. Research subjects reported awareness of physical problems ranging from physical weakness through to non-specific feelings of being unwell, as well as psychological problems like irritability, disinhibition or even paranoia. A few excerpts from the transcripts illustrate the finding:

1 Interviewer: Did you have weakness in your body?

ABI3: $\quad$ Yes just the left. It's still there. And it terrified my wife.

2 Interviewer: In terms of how you're doing after the injury, after the brain injury, what are you hoping for?

ABI2: $\quad$ To heal.

Interviewer: Heal?

ABI2: $\quad$ Yes, to heal up.

Interviewer: $\quad \mathrm{Ok}$, and what does that mean in specifics?

ABI2: $\quad$ The leg, the arm, the brain, the neck, the back, the mouth. ...the swearing.....the mood-control and behavior.

3 Interviewer: Yeah, what else changed that you weren't aware of? 
ABI5: $\quad$ That I wasn't aware of? Er... I suppose that...er........[long pause ].....how disinhibited I was.

4 ABI3: I get irritated quicker. Like "Oh, come on, let's go", you know? I'm like that, which isn't good.

Interviewer: Yeah. And that's following the sub-arachnoid hemorrhage?

ABI3: $\quad$ Yeah. I never used to be like that. I've got such a short temper.

5 ABI4: I've got a worse temper.

Interviewer: More of a temper?

ABI4: $\quad$ Well I used to have a bad temper before but I'd go all day long before I'd lose it - but now it's five minutes and it's gone, you know.

6 ABI2: I know I'm a bit crazy, paranoid about things like that. If you only knew the paranoia I go through in case like people stole my keys and put cameras in my workshop and things.

b) Metacognition: We encountered a similar result as regards metacognition, which has been defined as "knowledge or cognition about cognitive phenomena" (Flavell 1979) or "thinking about thinking" (Frith 2012). Despite the findings of the second research paradigm, we found that in the context of a capacity assessment interview, our patients manifested metacognitive abilities. Note that this evidence concerned both the patients' ability to think about their own psychological states and processes (excerpts $7,8,9,10$ ) and the ability to think about the psychological states of others (excerpts 10,11).

7 ABI4: Definitely. I'd find it harder to stick to stuff than what I did before. Before, if I decided something then I could stick to it 
quite well, but now when people start to explain this and that is better.....I find that hard to... Do you understand? ...I can't stick to things like I used to.

Interviewer: Yeah, I thought that might be. So there's this sort of sense that even when you think that something's a good idea, it's still difficult to commit to it?

ABI4: $\quad$ Even when you're adamant about something, it's too easy to opt out of it.

8 Interviewer: You were saying earlier that a lot of times things go over your head. What did you mean by that?

ABI2: $\quad$ I don't really sit and absorb what is actually happening.

9 ABI3: $\quad$ I tend to see things that I didn't see before, like beautiful flowers, when we're just driving along in the car, they may be on the side of the road or above a shop window. Things that I neglected before. I see those things now. Like beautiful sunsets, and sunrises...... [Inaudible.]

Interviewer: $\quad \mathrm{Mmmmm}$.

ABI3: $\quad$ And beautiful things like my grandchildren. Their smiling faces.

10 Interviewer: A new person. And you said at first you weren't aware there was a change.

ABI4: $\quad$ No I felt like myself but everything was different to friends. I didn't realize.....that I had actually changed. And when I realized that I had changed I moved away. My old mates....everyone knew me before, everyone knew me as I was.

11 Interviewer: And you know Dr F. - who's my colleague here - he's been listening in and thinking about what you've been saying. ABI2: $\quad$ He's probably been laughing at what a dickhead I am, really. 
c) Somatic Markers: Incorporating Bechara and Damasio's findings in the context of capacity assessment presents a number of its own distinctive challenges. As we have seen, the Somatic Marker Hypothesis combines both neurophysiological and psychological claims. Our research protocol did not incorporate neurophysiological monitoring, so we provide no data as regards the presence or absence of aSCRs in our research subjects. But as we have seen, such neurophysiological findings can have no direct application to legal assessment of capacity, since any legally attestable finding as regards DMC must be couched in psychological categories. Moreover, the specific findings in Bechara and Damasio's studies pertain to repeated iterations of a sequence of similar decisions undertaken in an experimental laboratory setting. aSCRs in the healthy population are learned responses after exposure to an iterated decision-task that provides immediate feedback as to benefit-or-loss. So while the neurophysiological results may be of considerable significance in advancing our understanding of the brain, they have no direct application in assessing the capacity of individual patients to make decisions outside the laboratory setting - particularly when such decisions may be one-off (whether to accept or refuse treatment) or where the balance of benefits and gains may manifest itself only over a long period (as with many disputed financial decisions). If the findings regarding somatic markers are to be made relevant to the assessment of DMC, then the task of "translation" must indeed be undertaken in earnest.

Our initial strategy was to take up Bechara and Damasio's own translation proposal, which turns on the hypothesis of a correlation between the neurophysiological deficit (absence of aSCRs) and a particular psychological deficit pertaining to the experience of emotion. One version of Bechara and Damasio's 
"translation" into psychological terms is framed as follows: "the abnormality is such that they do not engage emotions in relation to complex situations and events" (Bechara, Damasio et al. 2000, 295). Accordingly, we began by sorting the interview transcripts for traces of the presence or absence of this form of emotional engagement.

Our initial finding was that emotional engagement with complex situations and events was both reported and manifested in the interviews. Indeed it was not uncommon for the patients to be quite sentimental, and to manifest behavior patterns in which they sought emotional closeness with the interviewer.

12 ABI3: That's alright. I did tell you before that all you'd get is the truth out of me. I always tell the truth and I tell you my inner feelings as well, which is what you had some of today. And you're allowed to call me T., as well.

Interviewer: I can call you T.?

13 ABI6: Well you need people that care around you....you can't beat affection.

14 Interviewer: Yeah, you know, put a list. If someone asked you "What do you care most about?" what would you put at the top of the list?

ABI3: $\quad$ My wife and my family. My wife first, children and my grandchildren. And my Mum, rest her. My Mum was top of the tree, and my wife because I adore her.

15 ABI4: $\quad$ I used to have a really good support worker ... called S. We ended up really close, like best friends. ... I used to see him, he was like what they call a technical case manager.

Interviewer: He was like a support worker? 
ABI4: $\quad$ A case manager, yeah. I had two or three of them and then there was this bloke called $\mathrm{S}$, it was like a different association, and we were like really close and ... all my family loved him. I loved him, I thought he was brilliant. And then one day he died. And I was gutted.

These passages, and others like them, indicate that inability to engage emotionally with complex situations is not a well-represented deficit in our population of patients.

But the Somatic Marker Hypothesis is not a claim about emotionality or sentimentality as a general character trait. The phenomenon to which Bechara and Damasio have drawn attention is a particular function of a bodily response in influencing patient choices. Somatic markers do their work, according to Bechara and Damasio, by providing a kind of visceral signal to the decision-maker - a signal that operates independently of ordinary, discursive cognition. In one particularly striking result, research subjects with brain injuries continued to make the "wrong" (i.e., disadvantageous) bets even after reaching what Bechara and Damasio call "the conceptual stage" - i.e., a stage at which they could explain how the gambling task was structured and why the choices they were making were disadvantageous in the long run (Bechara et al. 1997). We therefore returned to the interview transcripts to look for traces of this phenomenon among our research subjects. We take up this theme further in $\S 5$, below; for now, we note that at least two of the research subjects did report a phenomenon that seems to reflect this form of non-discursive signaling. In both cases, the reports were offered in response to a series of questions about trust.

16 Interviewer: Tell me, when do you know whether something is threatening or not to be trusted? When do you know? How do you know? 
ABI2: $\quad$ I don't know, I just pick up on different things, like their body language, the way they are acting, what they're doing at the time, the things they say, plus people start lying....they might lie about something... and I might catch them out. And then I start questioning everything else they tell me and start looking into what they've told me before and then I find they're just lying about a lot of things

17 Interviewer: Yeah. Some people you feel comfortable with and trust, and others, as you were describing there, you don't at all. What makes the difference?

ABI3: $\quad$ It's a vibe. Now I trust you.

A pattern emerges from these initial results. The patients in our study each suffered from significant brain injuries. Family members and care-professionals expressed considerable doubt as to their competence to make decisions for themselves. But with our first attempt to translate results from the three research paradigms into strategies of investigation in the setting of a capacity interview, these patients systematically exhibited the range of abilities that have been shown to be negatively impacted in this patient population as a whole. If an assessor stopped at this point, $\mathrm{s} /$ he might well conclude that the presumption of capacity has not been overturned. But as we shall see, there proves to be more to the story.

\section{§5: A Modified Approach}

In getting beyond this initial obstacle, we found it useful to draw on an earlier research tradition which focused on the ways in which OPD patients navigate what we shall refer to as the decision situation. The starting point here is the phenomenological observation that decision-making is not a narrowly psychological 
or neurophysiological phenomenon. Decision-making does not take place "in the head"; it takes place in the world, and involves navigating a decision situation which is populated with options, opportunities, dangers, temptations, etc., as well as by the patient and other people. The ability to make decisions is therefore not simply a matter of understanding abstract information; it involves understanding the concrete decision situation, and finding a way to navigate it.

Nearly thirty years ago Lhermitte documented and described a distinctive dysfunction in the ways in which $\mathrm{ABI}$ patients respond to a decision situation. Patients suffering from environmental dependency syndrome (EDS) exhibit an "exaggerated dependency on the environment for behavioral cues" (Lhermitte 1986a, 326). One patient in Lhermitte's study would pick up a pair of spectacles laid on the table in front of him and attempt to put them on, even if he was already wearing glasses.

Lhermitte theorized EDS in both physiological and phenomenological terms. Physiologically, he proposed that EDS is associated with "liberation of parietal lobe activity, which is no longer submitted to the inhibitory effect of the frontal lobe" (Lhermitte 1986b, 142). Phenomenologically, he interpreted the syndrome as a disruption of the complex balance between engagement with and disengagement from one's immediate environment: “The shifting balance between dependence and independence with relation to the environment is one of the basic components of personal autonomy" (Lhermitte 1986b, 335).

Lhermitte's approach can itself be understood as an extension of an earlier research tradition associated with Goldstein. Drawing on his extensive interaction with soldiers who had suffered head injuries in the First World War, Goldstein developed a schema for evaluating ABI deficits. The schema reflects a 
phenomenologically informed attempt to articulate a set of abilities that comprise and sustain the individual patient's place in her world and to identify the ways that these abilities can be compromised in ABI. Goldstein drew particular attention to the distinction between what he called "the abstract and concrete attitudes," and to the danger in ABI of what he referred to as "the loss of the abstract attitude." In specifying the loss of the abstract attitude, Goldstein, with Sheerer, invoked the ability "to detach our ego from the outer world or from inner experience" (Goldstein and Sheerer 1941, 4). Some of their illustrative examples anticipate Lhermitte's results exactly, as in the case of a patient who, when asked to carry a comb across the room, could not do so without combing her hair.

In our attempt to "translate" recent research findings concerning ABI deficits into strategies for DMC-assessment, we were guided by this earlier, phenomenologically informed research tradition. None of the patients in our study exhibited the extreme forms of EDS documented by Lhermitte. Nonetheless, we found that his proposals concerning disruptions in the balance of engagement and disengagement with the decision situation were relevant to the difficulties faced by our research subjects. As we have seen, recent research has served to isolate certain well-defined patterns of deficit in the population of patients with injuries to the frontal lobe. But in assessing DMC, it is a strategic mistake to treat these deficits in isolation. Rather, the critical question is whether and how the ABI deficits serve to disrupt the balance between "the abstract and concrete attitude" or between engagement with and disengagement from the cues afforded by one's situation.

a) Awareness-of-Deficit and Metacognition: Consider first what this approach can show us about the interrelated phenomena of awareness-of-deficit and metacognition. Start with a series of exchanges with research subject ABI4. We have 
already seen that ABI4 exhibited both awareness of deficit (excerpt 5) and metacognitive abilities (excerpts 7 and 10). But these abilities were exhibited in isolation from the decision situations in which they might have been exercised. In following up with $\mathrm{ABI}$, we asked him to reflect on past decision-making episodes in particular the decisions associated with his discharge from hospital. Here a distinctive pattern came into view.

18 ABI4: It was really weird because in the hospital, everyone else was really bad. Like they couldn't walk or talk, totally mute ... . But I was fine, I was walking and talking, and I thought everything was fine. And when I went home, I was like gone. I couldn't walk, couldn't walk upstairs. I was stuck in bed, and I couldn't talk, you know... . It's nuts, isn't it? But when I was walking and talking I was fine. Every single day in the hospital I was asking if I could go home. "I want to go home, I want to go home. I'm fine, look at me. You can see I'm fine." So eventually they gave in and said "Go on then, go home". And once I was home it was just different, you know. Before that I'd felt like I was better, I was fine ... .

Interviewer: So when you were in hospital you felt it was all ok, you were walking around, you could speak, think, express yourself ...?

ABI4: $\quad$ I was feeling fine, ... but once I got out of hospital I realized how bad I was.

Interviewer: Outside of hospital it didn't work out?

ABI4: $\quad$ No, that's when I realized how bad I was.

Notice first that there is a sense in which this exchange itself exhibits both awareness-of-deficit and metacognition. Within the context of the interview situation, and hence retrospectively, ABI4 is showing awareness of some of the deficits 
associated with his injury and he is able to reflect on the process of thinking that led to his decision to be discharged. But the account he gives of the original decision situation itself is very different. In trying to orient himself in the decision situation, ABI4 lacked insight into his own deficits, and so was unable to incorporate information about his deficits in the decision about discharge. ABI4 was himself clearly aware, but only retrospectively aware, of this inability:

19 Interviewer: So this thing about not being able to see it at the time...not being able to see something at the time. I guess sometimes other people can see it at the time, but-

ABI4: Yeah if you're outside looking in you can see things, can't you, but on the inside you can't see it.

The exchange with ABI4 suggests a first refinement in the use of "awareness of deficit" as a category in assessment of DMC. At a minimum, we need to distinguish between retrospective and concurrent awareness-of-deficit. In order for awareness-of-deficit to inform the patient's navigation of the decision situation, it must be "real-time," - i.e., it must be concurrent with the deliberative process.

It is important to record one further observation about this exchange with ABI4. As the interviewer probed the patient's capacity to make decisions about place of residence, the patient himself shifted the modality of the exchange so that the interview itself took on the form of a decision situation.

20 ABI4: I want to get out, have a fresh start where no one knows me, and I don't know anybody, and start all over again. Start totally fresh, start a totally fresh life, a totally fresh life. 
Interviewer: And when you think like that, do you want to do it by yourself, alone, or do you want help from others?

ABI4: $\quad$ Do it myself.

Interviewer: Do it yourself?

ABI4: $\quad$ Yeah. I mean my uncle, when he got out of the nick ... 9 years or 10 years ... . He's out now, he's living up north ... . When I get out of hospital I could go and see him ... .

Interviewer: So what you're saying is that what you prefer is to start again, without any help from others. That's very, very different to hospital, isn't it, where there's an enormous amount of help that you're getting.

ABI4: I don't need this bollocks [hospital care], I'm sick of it.

As the interview shifts into a modality where the patient is once again deliberating about what to do, his awareness of deficit (and the associated recognition of the need for support) seems to dissipate. So even in the context of an interview in which he had shown awareness-of-deficit, the patient shows an inability to use that information to navigate the challenges of the decision situation. This in turn suggests that the relevant variable in awareness-of-deficit is not simply concurrence.

The importance of this point was illustrated emphatically in an exchange with another patient in the study:

21 Interviewer: I mean, for example, in the restaurant you had somebody kind of shout at you after you got irritated and you kind of got into an argument which had got a bit out of hand, and it sort of started because, whereas before you would have managed the situation, now you lose your temper?

ABI3: $\quad$ Yeah.

Interviewer: Can you think of examples like that? 
ABI3: $\quad$ Yeah it does happen. It does happen. [Noise from another patient in background.] I'll go out there and punch her on the fucking nose in a minute if she don't shut up!

In the context of this exchange, $\mathrm{ABI} 3$ shows explicit awareness-of-deficit, particularly as regards control of his temper. But even in the very act of expressing this awareness, he engages in the behavior that reflects the deficit. Concurrence in the awareness of deficit may well be a necessary condition for DMC, at least as regards certain classes of decision; but it is not a sufficient condition. For as ABI3 demonstrates rather vividly, concurrent awareness-of-deficit can fail to inform the patient's navigation of the decision situation. This is a phenomenon that we found reflected in other exchanges in the interviews, particularly with research subject ABI2:

22 ABI2: $\quad$ Yes. Again I do apologies for it [swearing/racist and sexist remarks]. I apologize every time - I spend two hours a day apologizing. I just get bored with it. I know it's outrageous and disgusting. I know it offends a lot of people but I just can't stop it.

23 Interviewer: Do you find certain things difficult to resist?

ABI2: $\quad$ Yeah, women and cars, and money and drugs, drink. All of those.

Interviewer: But for example, if a nice looking woman pulled up in a fancy car and said, you know, "Step in, but I want your £15,000” do you think you might hand it over?

ABI2: ...£150,000........£1.5 million ..... [inaudible] Interviewer: $\quad £ 15,000$.

ABI2: $\quad$ I don't know. She can have it, if she has a mini skirt and a nice peachy arse like Anna Kournikova, she could have $£ 150,000$ of mine if she wanted. 
Interviewer: But do you think that makes you a little bit vulnerable?

ABI2: $\quad$ In a way, I suppose - yeah it does.

Notice first that ABI2 clearly exhibits awareness-of-deficit and metacognition. He is aware of his inability to control his impulses, and he shows an ability to think about his own thinking retrospectively. What is striking is the extent to which these abilities are themselves disengaged from his behavior. It is as if his awareness of impulsivity was floating on the decision-making like a thin layer of foam on the surface of the sea. So what ABI2 lacks is not awareness-of-deficit or metacognition per se. What ABI2 lacks is effective online awareness-of-deficit and metacognition. Specifically, he lacks awareness-of-deficit and metacognition that is both (i) concurrent with episodes of decision-making and (ii) integrated into his navigation of the decision situation. (See Anderson and Lux 2004.)

b) Somatic Markers: We found that the concept of somatic markers was wellsuited to integration with the research tradition deriving from Lhermitte and Goldstein. Recall that somatic markers do their work precisely by influencing decisions, providing positive or negative "biasing" of some options over others. Otherwise put: the somatic markers serve to prefigure the decision situation, providing the decisionmaker with an essential orientation in his choice-environment.

We have documented above (excerpts $16 \& 17$ ) some exchanges in which our research subjects seemed to report traces of somatic prefiguring. But we also found evidence of the loss of this ability consequent upon $\mathrm{ABI}$.

24 ABI2: When I was younger, like about 15, if I hurt a woman I couldn't square .... I used to get a bad feeling in my heart, I just couldn't do it. Now - I was trying the other day - I don't get those 
feelings. I just say “cunt.” I just don't get any feeling that I'm being bad or naughty.

Notice that ABI2 is here reporting on the absence of a distinctive set of feelings that had once served to guide and constrain his behavior. In the language of traditional morality, we might think of this as a loss of conscience. But we should think of the operations of conscience broadly here: not simply as pronouncing moral judgment, either prospectively or retrospectively, but as mediating the individual's experience of a normatively complex world. ABI2 is not lacking in moral knowledge; retrospectively and discursively he is able to identify and articulate what was inappropriate in his behavior. But this normative orientation was absent in the distinctive modality where it matters most: in shaping his immediate sense for the normative complexities of his concurrent decision-situation. The result was that he systematically engaged in behavior that he only subsequently came to recognize as wrong. Similar reports were given by other subjects in the study.

25 Interviewer: Ok, so how do you check your own disinhibition, then, when you're making decisions? How do you check it?

ABI5: $\quad$ Er........ [long pause].... Er..... well I can't and I'm not aware until it's happened and er....and....

26 Interviewer: Things are different now?

ABI4 : $\quad$ I make the wrong choices without realizing it. ...it turns out..... [naudible] making the wrong choice.

Interviewer: So you can make the wrong choice without being aware that you're doing it?

ABI4 : $\quad$ And I don't realize until it's too late, you know. 
Taken together, this data suggests a distinctive but variable pattern of impairment in patients suffering from OPD. Some patients exhibit deficits in awareness-of-deficit, in metacognition and in somatic markers in ways that profoundly impoverished their sense for the normative texture of the decision situations with which they were faced. Some patients find themselves navigating high-stakes decision situations without the effective online metacognition, awareness of deficit, and implicit normative orientation on which they might ordinarily have relied. These forms of impairment can directly threaten DMC.

\section{§6: Conclusion: Decision-Making Abilities and Legal Attestability in ABI}

The concept of DMC has been defined in law in terms of a cluster of psychological abilities. These abilities must be jointly present in order for a patient to make his own decision. This construction of the legal concept of DMC is not simply a matter of convention; it is the principal strategy for avoiding an outcome test for capacity. Legally speaking, the question of capacity is not a question about what the patient decides, but about what the patient is able to do in reaching a decision.

In applying our findings to the task of capacity assessment, it is therefore useful to frame the results in the legally canonical idiom of abilities. Specifically, the data from our study serves to identify a set of three abilities that can be compromised in patients with $\mathrm{ABI}$ and OPD. Capacity-assessment interviews should therefore be designed to probe for these abilities, with respect to the decision that needs to be made. Insofar as these abilities are themselves essential components of DMC, evidence of their absence at the material time can constitute the basis for a finding of incapacity as regards a particular decision. The three abilities are as follows: 


\section{A. The ability to have 'real-time, online' awareness of deficits and}

impairments. The patients in our study often exhibited awareness of their own deficits, but this awareness was in several instances either unavailable to those patients in the decision situation, or else could not be integrated into the deliberative process. Assessors of DMC in this patient population should therefore not be satisfied by evidence of reflective awareness of deficit, but should probe for the ability to have effective, online awareness of deficits and awareness, as defined in $§ 5$, above. Where the patient's awareness of deficit cannot be integrated with the decision-making process, and where such awareness is essential for making the decision at hand (for example, as regards a proposed package of support arrangements), DMC can be undermined for that decision.

\section{B. The ability to detach from and engage with impulses and behavioral cues}

afforded by the decision situation. In any moderately complex decision situation, the decision-maker is faced with varieties of cues and stimuli to action. An individual with DMC characteristically has the ability (which he may or may not exercise) to step back from those cues and stimuli, temporarily disengaging his response in order to provide the time and space for deliberation. Several patients in our study either reported or exhibited an inability to suspend their behavioral responses in this way. For these patients, there is a class of decision-situations in which their response is effectively unmediated by reflection; in the limiting case it becomes little more than a motor response to an environmental stimulus. We also found evidence of a correlate inability: the inability to cut off the deliberative process in order to reengage with the immediate situation. One research subject reported on the way in which trivial decisions (e.g., about whether to have tea or hot chocolate) would lead him into cycles of paralyzing and irresolvable cycles of deliberation. In both cases, these inabilities 
threaten decision-making capacity, at least as regards the specific range of decision situations in which they are expressed.

C. The ability to prefigure a decision-situation by being attuned to relevant normative features. The ability to make decisions in a situation itself presupposes that the decision-maker either has or can acquire a sense of orientation in that situation, with a sense for the features and considerations that matter in reaching a decision. I can only 'use and weigh' information if I am alive to its normative significance. As the neurocognitive research has shown, our sense of orientation in a decision situation is produced in large part by processes that take place independently of conscious thought. For some of the patients in our study, this ability to find themselves normatively oriented in a decision situation is compromised, sometimes severely so. This is not to say that the decision-situation is normatively blank for them, but rather that important normative saliences of the situation come into view for these patients only after the decision has been made. In the words of ABI4: "I don't realize until it's too late." Where a patient is systematically blind to normative features of his situation that do indeed matter to him, he will need to be supported in ways that compensate for this inability. Where such support is unavailable or ineffective, DMC may be lost.

We can now return to the challenge of legal attestability. As noted at the outset, many of the behaviors associated with ABI and OPD are continuum phenomenon; i.e., they can be present or absent in varying degrees. Moreover, many of those behaviors can be found in one form or another in the healthy population. In order to arrive at a legally attestable assessment, we need to confront what we described earlier as the continuum and overlap problems. 
In addressing these problems, it is worth beginning with a conceptual point. In thinking about the continuum and overlap problems, it is tempting think that a solution will have to involve "drawing the line" at some specific point on the continuum. Since the relevant phenomena vary by degrees, it may seem as if we must find some precise value which sharply separates capacity from incapacity. Once framed in these terms, the problem can seem insuperable. How can one nonarbitrarily make such a demarcation? And will this not inevitably leave us with two individuals whose psychological abilities vary only minutely, but who find themselves on opposite sides of the line? How could we justify such an outcome?

But this way of framing the continuum and overlap problems is misleading. To begin with, it overlooks the importance of the legal principle of the presumption of capacity. (See, for example, MCA §1.2.) In the context of an adult assessment, the law requires the assessor to operate with the rebuttable presumption that the individual in question has DMC. A finding of incapacity must be based on the provision of evidence sufficient to overturn the presumption. This gives a rather different conceptual shape to the continuum and overlap problems. The task is not to discover the exact dividing line which separates the capacitous from the incapacitous population. Rather, the task is to determine whether, in a particular case, there is sufficient evidence to overturn the presumption of capacity. There may indeed be difficult cases or grey areas where such evidence is lacking. If so, then in those cases, the presumption is not overturned. So in dealing with the continuum and overlap problems, we should be looking for patterns of evidence with regard to particular capacities that suffice for overturning the presumption. This is still a hard problem in many cases, but it is not an impossible one, and it admits of non-arbitrary solutions. 
Here a second point becomes relevant: behaviors that manifest themselves along a continuum can themselves reflect underlying differences in kind. Consider the case of impulsivity. The assessor's task is not simply to determine the degree of impulsivity in a particular patient; this is at most a starting point for investigation. The task is to probe for the underlying source of the impulsive behaviour - whatever its degree. The data from the present study show that at least in some cases, impulsive behaviour in an $\mathrm{ABI}$ patient is itself the behavioral manifestation of certain underlying inabilities. In such cases the degree of impulsive behaviour may be high or low; this is in part a function of the environment in which the patient finds himself, the strength of behavioral cues, and many other factors. The crucial point is that for some patients, that impulsive behaviour is born of underlying inabilities that themselves render decision-making impossible, at least for certain classes of decision.

Consider how this applies to the assessment of ABI2's DMC with regard to financial decision-making. ABI2 makes impulsive financial decisions, as we know both from consulting informants and from the evidence he himself provides in the interviews. But impulsivity itself does not suffice to establish incapacity. What we are now in a position to add is that ABI2 lacks Ability B with respect to certain decision-situations. ABI2's impulsivity is the visible manifestation of an underlying inability: when faced with certain kinds of behavioral cues, he lacks the ability to disengage for the purposes of reflection. We can attest to this inability on the basis of what $\mathrm{ABI} 2$ himself tells us about past episodes of decision-making. With other patients in the study, the same inability was exhibited in the conduct of the interview itself. This form of impulsivity is not simply a difference in degree from the impulsivity found in the general population; it is different in kind. In confronting certain decision-situations, $\mathrm{ABI} 2$ simply lacks the ability to create the time and space 
for reflection; he lacks the ability to "engage the clutch" (so to speak) before responding. But without that ability he lacks the ability to deliberate ("use and weigh") in such circumstances; he therefore lacks DMC for that class of decisions. Rousseau famously argued that the natural roots of human freedom lie in a distinctive looseness in the connection between human instinct and human action (Rousseau 1755). Rather than responding immediately to stimuli with a motor response, human beings are possessed of a distinctive ability, at least in certain domains and within limits, to suspend the motor response in order to reflect on whether and how to react. Rousseau recognized that we do not always exercise this ability; in many instances we simply respond to the affordances of our situation. But the ability to disengage, and then to re-engage, is an important component of the phenomenological and psychological structure of decision-making. Indeed, we can say more: it is a condition on its very possibility. What Rousseau did not know is that certain forms of brain injury can compromise this distinctive human endowment. Where the ability to strategically engage and disengage is lost, the possibility for deliberation goes with it. And where there is no ability to deliberate, there is no DMC either. 
Acknowledgements

We thank the participants and staff that made the study possible. Support for the research was provided by the Wellcome Trust and the Arts and Humanities Research Council. We would like to thank David Batho, Matt Burch, Darren Carr, John Cutting, Anthony David, Simon Fleminger, Béatrice Han-Pile, Matthew Hotopf, Denzil Lush, Genevra Richardson, Alex Ruck Keene. Thanks also to two anonymous referees. 


\section{References}

Anderson, J., and W. Lux. 2004. Knowing your own strength: Accurate selfassessment as a requirement for personal autonomy. Philosophy, Psychiatry, \& Psychology 11(4): 279-294.

Bach, L. J., and A.S. David. 2006 Self-awareness after acquired and traumatic brain injury. Neuropsychological Rehabilitation 16(4): 397-414.

Bechara, A., A. Damasio, H. Damasio, and S.W. Anderson. 1994. Insensitivity to future consequences following damage to human prefrontal cortex. Cognition $50(1-3): 7-15$.

Bechara, A., D. Tranel, H. Damasio, and A. Damasio. 1996. Failure to respond autonomically to anticipated future outcomes following damage to prefrontal cortex. Cerebral Cortex 6(2): 215-225.

Bechara, A., H. Damasio, D. Tranel, and A. Damasio. 1997. Deciding Advantageously Before Knowing the Advantageous Strategy. Science 275(5304): 1293-1295.

Bechara, A., H. Damasio, D. Tranel, and S.W. Anderson. 1998. Dissociation of working memory from decision making within the human prefrontal cortex. The Journal of Neuroscience 18(1): 428-437.

Bechara, A., H. Damasio, A. Damasio, and G.P. Lee. 1999. Different contributions of the human amygdala and ventromedial prefrontal cortex to decision-making. The Journal of Neuroscience 19(13): 5473-5481.

Bechara, A., H. Damasio, and A. Damasio. 2000. Emotion, decision making and the orbitofrontal cortex. Cerebral Cortex 10(3): 295-307.

Bechara, A., and M. Van Der Linden. 2005. Decision-making and impulse control after frontal lobe injuries. Current opinion in neurology 18(6): 734-739. 
Charland, L. 2006. Anorexia and the MacCAT-T test for mental competence:

Validity, value, and emotion. Philosophy, Psychiatry, \& Psychology 13(4): 283287.

Charmaz, K. 2006. Constructing grounded theory: A practical guide through qualitative analysis. London: Sage Publications ltd.

Ciurli, P., U. Bivona, C. Barba, G. Onder, D. Silvestro, E. Azicnuda, J. Rigon, and R. Formisano. 2010. Metacognitive unawareness correlates with executive function impairment after severe traumatic brain injury. Journal of the International Neuropsychological Society 16(2): 360-368.

Craigie, J. 2009. Competence, practical rationality, and what a patient values. Bioethics 25(6): 326-333.

Damasio A. 1994. Descartes' error: Emotion, reason and the human brain. New York: Putnam Publishing.

Dunn, L. B., B.W. Palmer, and J. H. Karlawish. 2007. Frontal dysfunction and capacity to consent to treatment or research: Conceptual considerations and empirical evidence. In The Human Frontal Lobes: Functions and Disorders, eds. B. Miller and J.L. Cummings, 335-344. New York: The Guilford Press.

Flavell, J. H. 1979. Metacognition and cognitive monitoring: A new area of cognitive-developmental inquiry. American psychologist 34(10): 906-911.

Freedman, M., D.T. Stuss, and M. Gordon. 1991. Assessment of competency: the role of neurobehavioral deficits. Annals of internal medicine 115(3): 203-208.

Frith, C. D. 2012. The role of metacognition in human social interactions. Philosophical Transactions of the Royal Society B: Biological Sciences 367(1599): 2213-2223. 
Goldstein, Kurt, and M. Scheerer. 1941. Abstract and concrete behavior an experimental study with special tests. Psychological monographs 53(2): 1-151.

Grisso, T., \& Appelbaum, P. 1998. Assessing Competence to Consent to Treatment: A Guide for Physicians and Other Health Professionals. Oxford: Oxford University Press.

Hanten, G., M. Bartha, and H. S. Levin. 2000. Metacognition following pediatric traumatic brain injury: a preliminary study. Developmental Neuropsychology 18(3): 383-398.

Lhermitte, F., B. Pillon, and M. Serdaru. 1986a. Human autonomy and the frontal lobes. Part I: Imitation and utilization behavior: a neuropsychological study of 75 patients. Annals of neurology 19(4): 326-334.

Lhermitte, F. 1986b. Human autonomy and the frontal lobes. Part II: patient behavior in complex and social situations: the "environmental dependency syndrome". Annals of neurology 19(4): 335-343.

Martin, W. 2015. The MCA under scrutiny: Meeting the challenge of CRPDcompliance. Elder Law Journal 5(1):1-12

Masterman-Lister v. Brutton and Co [2002] EWCA Civ 1889.

Mental Capacity Act (England and Wales) 2005, available online at http://www.legislation.gov.uk/ukpga/2005/9/contents

Owen, G. S., F. Freyenhagen, M. Hotopf, and W. Martin. 2015. Temporal inabilities and decision-making capacity in depression. Phenomenology and the Cognitive Sciences 14(1): 163-182.

Ownsworth, T., and J. Fleming. 2005. The relative importance of metacognitive skills, emotional status, and executive function in psychosocial adjustment following 
acquired brain injury. The Journal of head trauma rehabilitation 20(4): 315332.

Prigatano, G. P. 1992. Personality disturbances associated with traumatic brain injury. Journal of Consulting and Clinical Psychology, 60(3): 360-368.

Reid-Proctor, G. Michelle, K. Galin, and M.A. Cummings. 2001. Evaluation of legal competency in patients with frontal lobe injury. Brain Injury 15(5): 377-386.

Rousseau, Jean-Jacques. 1755. Discourse on the Origins of Inequality. Amsterdam: Rey.

Schindler, B.A., D. Ramchandani, M.K. Matthews, and K. Podell. 1995. Competence and the frontal lobe: The impact of executive dysfunction on decisional capacity. Psychosomatics 36(4): 400-404.

Smith, J.A., P. Flowers, and M. Larkin. 2009. Interpretative Phenomenological Analysis: Theory Method and Research. London: SAGE Publications Ltd.

Tan, J., A. Stewart, R. Fitzpatrick, and R.A. Hope. 2006. Competence to make treatment decisions in anorexia nervosa: thinking processes and values. Philosophy, Psychiatry, \& Psychology 13(4): 267-282. 